\title{
Electronic structure of Fibonacci Si $\delta$-doped GaAs
}

\author{
F. Domínguez-Adame, E. Maciá*, and B. Méndez \\ Departamento de Física de Materiales, Facultad de Físicas, Universidad Complutense, E-28040 \\ Madrid, Spain
}

(February 6, 2008)

\begin{abstract}
We study the electronic structure of a new type of Fibonacci superlattice based on Si $\delta$-doped GaAs. Assuming that $\delta$-doped layers are equally spaced, quasiperiodicity is introduced by selecting two different donor concentrations and arranging them according to the Fibonacci series along the growth direction. The one-electron potential due to $\delta$-doping is obtained by means of the Thomas-Fermi approach. The resulting energy spectrum is then found by solving the corresponding effective-mass wave equation. We find that a self-similar spectrum can be seen in the band structure. Electronic transport properties of samples are also discussed and related to the degree of spatial localization of electronic envelope-functions.
\end{abstract}

PACS number(s): 71.25.-s, 73.61.Ey, 71.45.Jp 


\section{INTRODUCTION}

Following the first fabrication of quasiperiodic semiconductor superlattices [1,2], there has been a increasing interest in the study of one-dimensional systems describing quasiperiodic structures. From the very beginning, most researchers have considered the Fibonacci sequence as a typical example of a quasiperiodic system [3.4], and several characteristic properties of Fibonacci systems have been reported during the last decade. Thus, it is now well established that Fibonacci lattices exhibit highly fragmented electron and phonon spectra with a hierarchy of splitting subbands displaying self-similar patterns [5], and their corresponding electronic density of states shows spiky features [6]. This exotic electronic spectrum strongly influences electron propagation [0,8] and dc conductance through the system, even at finite temperature [9]. Furthermore, electronic wave functions are neither extended, in the Bloch sense, nor exponentially localised; instead, they are critical in Fibonacci lattices [10].

All these striking results, however, have been obtained for two particular kinds of model Hamiltonians, namely tight-binding models (either diagonal, off-diagonal or both) and Kronig-Penney models. Therefore, the question as to whether those features purported so far as characteristic of Fibonacci order are extensible to more realistic Fibonacci Hamiltonians becomes very appealing from a theoretical point of view. On the other side, since it was realized that Fibonacci superlattices (FSLs) preserve its quasiperiodic order even if substantial growth fluctuations in the sequential deposition of layers is allowed for [2], a considerable interest on the transport properties of quasiperiodic semiconductor heterostructures has emerged in the experimental field. However, FSLs studied up to date are quantum-well GaAs/AlAs based ones. This shortcoming does not seem necessary from an experimental viewpoint, since computer controlled doping deposition techniques are currently able to construct a wide variety of superlattice potential profiles. In particular, epitaxial-growth techniques allow to prepare $\delta$-doped semiconductor structures, in which a sheet of donors is localized within a few monolayers of the crystal. These impurity atoms supply electrons and give rise to strong confinement by the resulting one-electron potential.

The main purpose of this letter is to show that distinctive features, previously obtained for simpler Fibonacci Hamiltonians, also appear in more complex and realistic systems, hence strongly suggesting that those features can be a universal fingerprint of one-dimensional Fibonacci systems. To this end, in the present work we propose a new type of FSL based on quasiperiodically $\mathrm{Si} \delta$-doped GaAs. We study the resulting electronic structure within the envelope-function and effective-mass approximation. The one-electron potential is found by solving the Thomas-Fermi equation. We find that the electronic spectrum is highly fragmented and shows self-similar patterns which become very sensitive to the doping ratio concentration. The spectral type of our system is analysed by means of bandwidth-scaling techniques suggesting a underlying singular continuous character. Finally, transport properties of the FSL at zero temperature are discussed in the context of the Landauer formula and related to the possible critical nature of the electronic states. 


\section{THE MODEL}

The system we study in this work is a FSL made of $\mathrm{Si} \delta$-doped GaAs. In general, a FSL of order $N$ is generated from two basic units $\mathrm{A}$ and $\mathrm{B}$ by successive applications of the inflation rule $\mathrm{A} \rightarrow \mathrm{AB}$ and $\mathrm{B} \rightarrow \mathrm{A}$ yielding a sequence of the form ABAABABA $\ldots$ This sequence comprises $F_{N-1}$ elements $\mathrm{A}$ and $F_{N-2}$ elements $\mathrm{B}, F_{l}$ being the $l$ th Fibonacci number given by the recurrent law $F_{l}=F_{l-1}+F_{l-2}$ with the initial values $F_{0}=F_{1}=1$. In the present model we take each unit (A or B) as a slab of GaAs of thickness $a=200 \AA$ with a Si $\delta$-doped layer embedded in its center. The quasiperiodicity is then introduced by allowing the doping layers to take on two possible values of donor concentration, and arranging them according to the Fibonacci sequence. Each doping layer consists of a continuous positive slab of thickness $d=50 \AA$ with either $N_{D}^{(A)}$ or $N_{D}^{(B)}$ ionized donors per unit area. Hereafter we fix $N_{D}^{(A)}=5 \times 10^{12} \mathrm{~cm}^{-2}$, and allow $N_{D}^{(B)}$ to vary from 2.0 up to $6.5 \times 10^{12} \mathrm{~cm}^{-2}$ in order to study the influence of the doping ratio $r \equiv N_{D}^{(B)} / N_{D}^{(A)}$ onto the resulting electronic spectrum. Finally, we assume that there exists a uniform p-type background doping with $N_{A}=10^{15} \mathrm{~cm}^{-3}$ acceptors per unit volume. This range of parameters corresponds to samples which could be easily grown by molecular beam epitaxy techniques now available.

First we compute the one-electron potential $V_{j}(x)$ in each basic unit (here $j$ denotes A or $\mathrm{B}$ ), where $x$ indicates the spatial coordinate along the growth direction. This we accomplish by means of the Thomas-Fermi (TF) semiclassical model. It is known that the $\mathrm{TF}$ formulation is equivalent to the self-consistent formulation in a wide range of doping concentrations [1], and it has been successfully applied to periodically Si $\delta$-doped GaAs SLs [12 14]. Hence we can confidently adopt this approach to calculate the one-electron potential due to the layer of ionized donors. Choosing the middle of the $\delta$-doped layer as the origin of coordinates, the nonlinear $\mathrm{TF}$ differential equation reads

$$
\begin{aligned}
\frac{d^{2} V_{j}(x)}{d x^{2}} & =-\frac{8}{3 \pi}\left[\epsilon_{F}-V_{j}(x)\right]^{3 / 2}+\frac{8 \pi}{d} N_{D}^{(j)} \theta\left(\frac{d}{2}+x\right) \theta\left(\frac{d}{2}-x\right) \\
& -8 \pi N_{A} \theta\left(\frac{a}{2}+x\right) \theta\left(\frac{a}{2}-x\right), \quad j=\mathrm{A}, \mathrm{B},
\end{aligned}
$$

where $\epsilon_{F}$ denotes the Fermi energy. The value of the Fermi energy cannot be computed self-consistently in the $\mathrm{TF}$ formulation, so it must be regarded as a parameter. Thus we fix the origin of energies so $\epsilon_{F}=0$ henceafter. Distances and energies are scaled in units of the effective Bohr radius $\mathrm{a}^{*}(=100 \AA)$ and effective Rydberg $\mathrm{Ry}^{*}(=5.8 \mathrm{meV})$, respectively. The boundary conditions for this equation are those of the superlattice given by [12] $V_{j}^{\prime}(0)=V_{j}^{\prime}(a / 2)=0$. The resulting potential becomes deeper in the gap on incresing donor concentration.

We assume the validity of the effective-mass approximation, and we take isotropic and parabolic conduction band in the growth direction. This approach works well in some directgap compounds, as it is the case of GaAs. Once the potential $V_{j}(x)$ in each basic unit is found from (1), the electron envelope-function and energy values can be obtained from the following one-dimensional Schrödinger equation

$$
-\frac{d^{2} \psi(x)}{d x^{2}}+V(x) \psi(x)=E \psi(x)
$$


where $V(x)$ is the potential of the whole FSL. We assume that this potential at any point of the system is given by the one-electron potential $V_{j}(x)$ we have just computed in each basic unit, where $V_{A}$ and $V_{B}$ are arranged according to the Fibonacci series. Therefore, $V(x)$ is simple a piecewise potential with $F_{N}$ regions.

\section{NUMERICAL ANALYSIS}

Since there is no analytical expression for the potential $V_{j}(x)$, computations must rely on numerical procedures. To this end, we divide the each basic unit of the FSL in a grid of points $\left\{x_{k}=k h\right\}$, where $h=a / n$ is the integration step and $n$ is the number of grid points in each basic unit. In our calculations we have taken $n=400$, which is more than enough to obtain very accurate results. The discretized form of the Schrödinger equation (2) may be cast in the matrix form

$$
\left(\begin{array}{c}
\psi\left(x_{k+1}\right) \\
\psi\left(x_{k}\right)
\end{array}\right)=\left(\begin{array}{cc}
\alpha_{k} & -1 \\
1 & 0
\end{array}\right)\left(\begin{array}{c}
\psi\left(x_{k}\right) \\
\psi\left(x_{k-1}\right)
\end{array}\right) \equiv P_{k}\left(\begin{array}{c}
\psi\left(x_{k}\right) \\
\psi\left(x_{k-1}\right)
\end{array}\right),
$$

where we have defined $\alpha_{k} \equiv 2+h^{2}\left(V\left(x_{k}\right)-E\right)$ for brevity. Iterating this equation one obtains

$$
\left(\begin{array}{c}
\psi\left(x_{N_{S L}+1}\right) \\
\psi\left(x_{N_{S L}}\right)
\end{array}\right)=P_{N_{S L}} \cdots P_{0}\left(\begin{array}{c}
\psi\left(x_{0}\right) \\
\psi\left(x_{-1}\right)
\end{array}\right) \equiv T\left(N_{S L}\right)\left(\begin{array}{c}
\psi\left(x_{0}\right) \\
\psi\left(x_{-1}\right)
\end{array}\right)
$$

$T\left(N_{S L}\right)$ is the transfer matrix of the FSL and $N_{S L}=n F_{N}$ is the number of grid points in the whole structure. $T\left(N_{S L}\right)$ is real and relates the envelope-function at both edges of the structure. Taking into account that $T(k)=P_{k} T(k-1)$ and $T(0)=P_{0}$ we find the following recurrence relations involving only real parameters

$$
\begin{aligned}
& T_{11}(k)=\alpha_{k} T_{11}(k-1)-T_{11}(k-2), \\
& T_{12}(k)=\alpha_{k} T_{12}(k-1)-T_{12}(k-2), \\
& T_{21}(k)=T_{11}(k-1), \\
& T_{22}(k)=T_{12}(k-1), \quad k=1,2 \cdots N_{S L} .
\end{aligned}
$$

These equations must be supplemented with the initial conditions $T_{i j}(-1)=\delta_{i j}, T_{11}(0)=\alpha_{0}$, $T_{12}(0)=-1, T_{21}(0)=1$ and $T_{22}(0)=0$. The resulting allowed energies can be found by imposing periodic boundary at both edges of the system with $F_{N}$ layers arranged according to the Fibonacci sequence. Once we have obtained the transfer matrix for the whole FSL, the resulting energies for which the absolute value of the trace of the transfer-matrix corresponding to the whole FSL is smaller (larger) than 2 are allowed (forbbiden) [15].

\section{RESULTS AND DISCUSSIONS}

In order to properly discuss the novel features arising from quasiperiodicity in our system, it is convenient to give a brief account of the electronic spectrum associated to this model when all $\delta$ layers have the same donor concentration. In this case, which corresponds to a 
usual periodic superlattice, resonant coupling between identical states of neighbouring layers leads to the formation of minibands of finite width [12 14, related to extended (Bloch type) electronic states. The resulting miniband structure and the dispersion relation inside allowed minibands $E(K), K$ being the momentum perpendicular to the layers, can be evaluated using the transfer-matrix approach as well. Since Bloch theorem must be satisfied in the periodic case, the dispersion relation is found to be (see Ref. [16] for details)

$$
\cos (K a)=\frac{1}{2} \operatorname{Tr}[T(n)]
$$

Notice that the required time-reversal symmetry of the dispersion relation $E(-K)=E(K)$ is conserved. As an illustration, we show in Figure 1 the miniband structure for a particular realization corresponding to a donor concentration of $5 \times 10^{12} \mathrm{~cm}^{-2}$. Note that the lower miniband is almost nondispersive since its width is rather small (about 0.4 Ry*). This suggests that the ground state of the Thomas-Fermi potential is only weakly coupled to its neighbouring wells. On the contrary, the second miniband is clearly dispersive, as seen in Fig. 1, and its width amounts $4.7 \mathrm{Ry}^{*}$. The third miniband is even wider and it crosses the Fermi level.

Now, we consider the most prominent features of the resulting electronic structure when quasiperiodicity is introduced. A schematic diagram of the FSL potential for $F_{5}=8$ is presented in Fig. 2. From a mathematical point of view, one of the most characteristic properties of electronic spectra in Fibonacci systems is its highly fragmented, Cantor-like nature. We have confirmed this fragmentation in our FSL even when deviation from perfect periodicity is actually small, in other words, when the ratio $r$ is close to unity. In fact, we have found that each miniband of the periodic SL, shown in Fig. 1, splits in several sub-minibands, that is, small gaps appear. The origin of these small inner gaps are directly related to the loss of long-range quantum coherence of the electrons, as the potential inside basic units, A and B, becomes different. Results corresponding to the fragmentation of the second miniband are shown in Fig. 3 as a function of the Fibonacci order $N$. We have mainly focused on this second miniband since the first one is almost nondispersive and investigation of the fragmentation process would require very tiny energy steps and then is rather time consuming. Only short approximants of the FSL are displayed in Fig. 3 since on increasing $N$ the spectrum becomes so fragmented that it is difficult to observe minor features in the plot. However, we have carefully analyzed FSLs spectra up to order $N=12$ (233 layers) and we have confirmed that the number of sub-minibands composing the whole spectrum is exactly $F_{N}$, i.e. the number of basic units forming the superlattice.

As we mentioned in the Introduction, another characteristic feature of Fibonacci systems is the self-similar pattern exhibited by their corresponding spectra. This self-similarity has been widely investigated within the tight-binding approximation while much less work has been devoted to quantum-well superlattices. Our results show that self-similar spectra are also obtained in Fibonacci Si $\delta$-doped GaAs, as shown in Fig. 4 . It is clear that the whole electronic spectrum for a short approximant $\left(F_{4}=5\right.$ in this case) is mapped onto a small portion of the spectrum of a higher approximant $\left(F_{7}=21\right.$ in Fig. 4). This is a consequence of how the FSL is constructed, based on a deterministic substitution sequence [17].

A third characteristic of Fibonacci systems concerns their spectral type, which results to be singular continuous. In order to estimate the spectral type associated to our model 
Hamiltonian, we have calculated the normalized equivalent bandwidth $S$, defined as the ratio between the sum of all allowed sub-minibands and the width of the second miniband in the periodic $A$ SL $\left(4.7 \mathrm{Ry}^{*}\right)$. As can be expected from the Cantor-like nature of Fibonaccian spectra, $S$ vanishes as the system size grows. Furthermore, we have obtained that the normalized equivalent bandwidth decreases according to a power law of the form $S=F_{N}^{-\beta}$ with $\beta \sim 0.1$, as seen in Fig. 0 . According with earlier works [17], such a behaviour is characteristic of a singular continuous spectrum for which all the envelope-functions are critical, i.e., regarding localization properties the functions are neither exponentially localized nor extended.

The richness in structure displayed by the electronic spectrum should be reflected, to some extent, in its transport properties [9]. We have evaluated the electrical resistance at zero temperature, $\rho$, using the well-known dimensionless single-channel Landauer formula [18]: $\rho=R\left(\epsilon_{F}\right) / T\left(\epsilon_{F}\right)$, where $R$ and $T$ denote the reflection and transmission coefficients, respectively. The transmission coefficient can be obtained in a straightforward manner in the transfer-matrix formalism [16]. A typical example of the obtained results is shown in Fig. 6(a) for $F_{11}=144$. We observe that the Landauer resistance exhibits a highly fragmented structure displaying dramatic fluctuations under minor variations of the ratio $r$. The practical implications of such behaviour should be clear: One can select almost any desired value of the resistance of the sample by choosing properly the suitable ratio $r$. According to previous works [9], on increasing temperature interesting behaviours of the resistance can be expected since the detailed structure of the energy spectrum naturally determines the finer details of the resistance pattern at finite temperature.

We have also investigated the spatial extent of the electron envelope-functions at the Fermi level. This we accomplished by means of the inverse participation ratio (IPR), as defined, for instance, in Ref. [6]

$$
\mathrm{IPR}=\frac{\sum_{k}\left|\psi\left(x_{k}\right)\right|^{4}}{\left(\sum_{k}\left|\psi\left(x_{k}\right)\right|^{2}\right)^{2}},
$$

where the index $k$ runs over grid points of the whole FSL. The IPR gives an estimation of the volume occupied by the electron envelope-function: The smaller the IPR, the more extended the electron state. Although a more detailed analysis of the envelope-functions is required to determine the exact nature of the wave function (multifractal analysis), for an understanding of the resistance behaviour the IPR is sufficient. Figure 6(b) shows the IPR for $F_{11}=144$ as a function of the ratio $r$. Note that the minimum value is reached when this ratio becomes unity, i.e. in the periodic superlattice. The IPR also present dramatic fluctuations under minor variation of the donor concentration $N_{D}^{(B)}$. A comparison of both Fig. 6(a) and Fig. 6(b) reveals that the IPR is larger (electronic states are more localized) whenever the resistance increases, indicating that the spatial extent of the envelope-functions controls the electrical transport of the sample.

\section{CONCLUSIONS}

We have proposed a new type of quasiperiodic (Fibonacci) superlattice heterostructure based on $\mathrm{Si} \delta$-doped GaAs. The corresponding electronic spectrum shows a highly fragmented, self-similar nature resembling that found for simpler tight-binding models. The 
spectral type of our model Hamiltonian, obtained from bandwidth-scaling considerations, indicates that it is singular continuous in the thermodynamical limit, in agreement with the current opinion of the mathematical community, supporting the conjecture that the spectral type for almost all substitution sequences should be singular continuous [19. The spectrum structure is very sensitive to the doping difference between the basic units, and this fact significantly affects the transport properties of the sample at zero temperature. This interesting result suggests the possibility of a certain degree of "engineering" of transport properties during superlattice growth by a proper selection of the corresponding doping sequences. Finally, we are able to relate resistance fluctuations to the nature of the electronic wave function, through the inverse participation ratio. The obtained relationship suggests that the overall conductance of the superlattice is directly connected with the decay rate of the electron wave function along the sample. In this sense, it would be convenient to perform

a complete analysis of the wave function espatial distribution by means of the multifractal formalism. Work in this direction is currently in progress and we expect to report on it elsewhere.

\section{ACKNOWLEDGMENTS}

The authors thank A. Sánchez for a critical reading of the manuscript. This work has been partially supported by Univeridad Complutense under project PR161/93-4811. 


\section{REFERENCES}

* Also at the Instituto de Estudios Interdisciplinares, El Guijo, Z4 Galapagar, E-28260 Madrid, Spain.

[1] R. Merlin, K. Bajema, R. Clarke, F. -Y. Juang, and P. K. Bhatacharya, Phys. Rev. Lett. 55, 1768 (1985).

[2] J. Todd, R. Merlin, R. Clarke, K. M. Mohanty, and J. D. Axe, Phys. Rev. Lett. 57, 1157 (1986).

[3] M. Kohmoto, L. P. Kadanoff, and C. Tang, Phys. Rev. Lett. 50, 1870 (1983).

[4] S. Ostlund and R. Pandit, Phys. Rev. B 29, 1394 (1984).

[5] F. Laruelle and B. Etienne, Phys. Rev. B 37, 4816 (1988).

[6] K. Hirose, D. Y. K. Ko and H. Kamimura, J. Phys.: Condens. Matter 4, 5947 (1992).

[7] S. Katsumoto, N. Sano, and S. Kobayashi, Solid State Commun. 85, 223 (1993).

[8] F. Domínguez-Adame and A. Sánchez, Phys. Lett. A 159, 153 (1991).

[9] E. Maciá, F. Domínguez-Adame, and A. Sánchez, Phys. Rev. B 49, 9503 (1994).

[10] A. Chakrabarti, S. N. Karmakar, and R. K. Moitra, Phys. Lett. A 168, 301 (1992).

[11] L. Ioriatti, Phys. Rev. B 41, 8340 (1990).

[12] J. C. Egues, J. C. Barbosa, A. C. Notari, P. Basmaji, L. Ioriatti, E. Ranz, and J. C. Portal, J. Appl. Phys. 70, 3678 (1991).

[13] B. Méndez and F. Domínguez-Adame, Phys. Rev. B 49, 11471 (1994).

[14] F. Domínguez-Adame, B. Méndez, and E. Maciá, Semicond. Sci. Technol. 9, 263 (1994).

[15] J. M. Ziman, Models of Disorder (Cambridge University Press, London, 1979).

[16] B. Méndez, F. Domínguez-Adame, and E. Maciá, J. Phys. A: Math. Gen. 26, 171 (1993).

[17] M. Kohmoto, Phys. Rev. Lett. 51, 1198 (1983).

[18] R. Landauer, IBM J. Res. Dev. 1, 223 (1957).

[19] A. Bovier and J. -M. Ghez, Commun. Math. Phys. 158, 45 (1993). 


\section{FIGURES}

FIG. 1. Miniband structure for periodically $\mathrm{Si} \delta$-doped GaAs with a donor concentration $5 \times 10^{12} \mathrm{~cm}^{-2}$.

FIG. 2. Schematic diagram of the one-electron potential for a Fibonacci Si $\delta$-doped GaAs with $N_{D}^{(A)}=5 \times 10^{12} \mathrm{~cm}^{-2}$ and $N_{D}^{(B)}=4.5 \times 10^{12} \mathrm{~cm}^{-2}$ with $N=F_{5}=8$ layers.

FIG. 3. Allowed sub-minibands as a function of the Fibonacci order $N$, for a Fibonacci Si $\delta$-doped GaAs with $N_{D}^{(A)}=5 \times 10^{12} \mathrm{~cm}^{-2}$ and $N_{D}^{(B)}=4.5 \times 10^{12} \mathrm{~cm}^{-2}$. The number of sub-minibands is $F_{N}$ for each order $N$.

FIG. 4. Self-similar spectrum of a Fibonacci Si $\delta$-doped GaAs with $N_{D}^{(A)}=5 \times 10^{12} \mathrm{~cm}^{-2}$ and $N_{D}^{(B)}=4.5 \times 10^{12} \mathrm{~cm}^{-2}$. Left plot shows the whole spectrum of a FSL of order $N=4$ whereas right plot shows a detail of the spectrum of the FSL of order $N=7$.

FIG. 5. Log-log plot of the normalized equivalent bandwidth $S$ as a function of the number of $\delta$-doped layers $F_{N}$ for a Fibonacci Si $\delta$-doped GaAs with $N_{D}^{(A)}=5 \times 10^{12} \mathrm{~cm}^{-2}$ and $N_{D}^{(B)}=4.5 \times 10^{12} \mathrm{~cm}^{-2}$.

FIG. 6. (a) Landauer resistance at the Fermi energy and (b) IPR as a function of the ratio $N_{D}^{(B)} / N_{D}^{(A)}$ for a Fibonacci Si $\delta$-doped GaAs with $N_{D}^{(A)}=5 \times 10^{12} \mathrm{~cm}^{-2}$ and $F_{11}=144$ layers. 